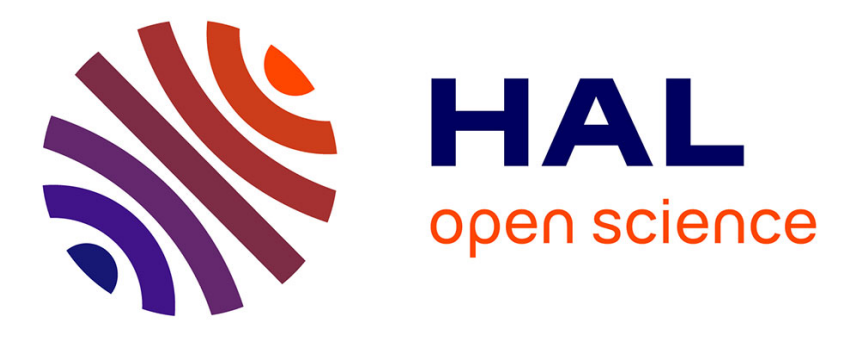

\title{
Time-resolved measurements from speckle interferometry
}

Jean-Michel Tualle, Ha Lien Nghiem, Christof Schäfauer, Patrice Berthaud, Eric Tinet, Dominique Ettori, Sigrid Avrillier

\section{- To cite this version:}

Jean-Michel Tualle, Ha Lien Nghiem, Christof Schäfauer, Patrice Berthaud, Eric Tinet, et al.. Timeresolved measurements from speckle interferometry. Optics Letters, 2005, Vol. 30 (1), pp.50-52. hal-00003782

\section{HAL Id: hal-00003782 \\ https://hal.science/hal-00003782}

Submitted on 5 Jan 2005

HAL is a multi-disciplinary open access archive for the deposit and dissemination of scientific research documents, whether they are published or not. The documents may come from teaching and research institutions in France or abroad, or from public or private research centers.
L'archive ouverte pluridisciplinaire HAL, est destinée au dépôt et à la diffusion de documents scientifiques de niveau recherche, publiés ou non, émanant des établissements d'enseignement et de recherche français ou étrangers, des laboratoires publics ou privés. 


\section{Time-resolved Measurements from Speckle Interferometry}

J.-M. Tualle, H.L. Nghiêm, C. Schäfauer, P. Berthaud, E. Tinet, D. Ettori and S. Avrillier Laboratoire de Physique des Lasers (CNRS UMR 7538),

Université Paris 13, 99 avenue J-B. Clément, 93430 Villetaneuse, France

\section{$\underline{\text { ABSTRACT }}$}

We present in this paper time-resolved measurements by Speckle Interferometry of the light scattered by a liquid medium. The measurements are performed using a reflectance geometry, and are compared to the results obtained in the same conditions using a femtosecond laser and a streak camera. The setup was also tested in vivo on the forearm of a volunteer, showing the potential of such a setup for biomedical applications.

( OCIS : $170.3660,170.6920,170.6480$ ) 
Biological tissues present a transmission window for near infrared wavelengths, which allows to probe optical properties deep inside the tissue. The measurement of such optical properties can bring a lot of useful information for medical diagnosis ${ }^{[1]}$. Such measurements however involve inverse problems due to the complex biological structures encountered in real life, and the use of time-resolved measurements of the scattered light can provide a lot of pertinent information to overcome those difficulties ${ }^{[2-6]}$.

Time-domain measurements however imply expensive and high-tech experiments, since the time resolution needed is in the ten picoseconds range. We have previously shown ${ }^{[7,8]}$ that the same information can be obtained by a simpler technique: Briefly, when a standard Mach-Zehnder interferometer with a propagation time difference $\delta \tau$ between its two arms, is illuminated by a frequency modulated $\mathrm{CW}$ source, with a radian frequency $\omega(t)=\omega_{0}+\Delta \Omega \cos (2 \pi f t)$ for a sinusoidal modulation, the recorded interference signal is proportional to $\cos \left[\Delta \Omega \delta \tau \cos (2 \pi f t)+\omega_{0} \delta \tau\right]$. Let us introduce the numerically generated function

$$
\operatorname{Ref}(t, \tau)=\sin ^{2 n}(2 \pi f t) \exp [i \Delta \Omega \tau \cos (2 \pi f t)]
$$

and the quantity $S_{D C}(\tau)$ defined as the average value over half a period of the product $s(t) \operatorname{Ref}(t, \tau)$. This quantity is the equivalent of what would be a Fourier transform for a linear modulation. We however choose to work with a sinusoidal modulation in order to limit potential problems that can arise from a limited bandwidth of the modulation system. It is straightforward to see ${ }^{[7]}$ that $\left|S_{D C}\right|^{2}(\tau) \propto g^{2}(\tau-\delta \tau)$, where the function $g^{2}$ acts as a time gate that is all the narrower since $\mathrm{n}$ is low, and that decreases all the faster since $\mathrm{n}$ is high. A good compromise is to set $n=2$, which allows to reach a $45 \mathrm{ps}$ time resolution with a $30 \mathrm{GHz}$ modehop free modulation $(\Delta \Omega=2 \pi 15$ 
GHz). The trick now is that if we insert a turbid medium in one arm in the interferometer, and perform an ensemble averaging, we obtain ${ }^{[7]}$ :

$$
\left\langle\left|S_{D C}\right|^{2}\right\rangle(\tau) \propto \int g^{2}\left(\tau-\tau^{\prime}\right) \phi\left(\tau^{\prime}\right) d \tau^{\prime}
$$

where $\phi$ is the light flux incident on the detector. We stress here that the detector can be larger than the coherence area, even if it doesn't increase the recorded signal.

We have already proved the feasibility of this method in a transmittance geometry through a solid turbid medium, where the ensemble averaging was performed by slightly moving the incident laser beam, but this method remained to be tested on liquid and biological media. This implied to increase the modulation frequency of the source (our first experiment was carried out with a modulation frequency of less than $1 \mathrm{~Hz}$ ), in order to freeze the fluctuations of the speckle pattern linked to microscopic movements inside the medium, and therefore to avoid decorrelation. In the same way, the sensitivity of the experiment had to be increased in order to compensate this reduction of the acquisition time.

Our new experimental setup is depicted in figure 1 . The wavelength modulated source is a Littman extended cavity TEC 500 from Sacher Lasertechnik, emitting $7 \mathrm{~mW}$ at $\lambda=780 \mathrm{~nm}$, with a line width of about $1 \mathrm{MHz}$. This source allows a modehop free modulation of about $30 \mathrm{GHz}$ at a frequency $f=300 \mathrm{~Hz}$. Using these values in (1), and for values of the time of flight $\tau$ that can reach $4 \mathrm{~ns}$, it appears that the detection system needs to have a bandwidth better than $100 \mathrm{kHz}$. Using PIN photodiodes S5972, we reached a bandwidth of $300 \mathrm{kHz}$ with a NEP of $230 \mathrm{fW} . \mathrm{Hz}^{-1 / 2}$. The high quantum efficiency of the photodiodes is required in order to achieve the best signal to noise ratio (SNR). Furthermore, in order to point out the fact that the detectors can be larger that the coherence area without any lowering of the SNR, the whole experiment was performed using 
graded-index multimode fibers for both the signal and the reference arms, the light from these fibers being of course completely collected by the detectors. The use of fibers allows to exploit different detection geometries, such as reflectance measurements. This system allows to reach a 50ps time resolution, due to a small dispersion in the multimode fibers.

Given the very low signal we have to measure, the main problem we had to face was a forest of parasitic signals from various origins. We carefully designed the experiment in order to cancel such unwanted signals using an optical isolator, a low reference signal ( $1 \mu \mathrm{W}$ in the following) and a balanced detection, but the only way we had found to remove this difficulty completely was to perform a periodic background subtraction: an acousto-optic modulator (AOM in figure 1) was placed in the signal arm, and the zeroth order is sent to the scattering medium. This allows to perform a measurement during a modulation period, followed on next period by a measurement at low signal: the difference between these measurements cancels the parasitic signals completely.

Our purpose was to test this setup on a liquid medium. Considering only Brownian motion, this implies ${ }^{[9]}$ for the modulation frequency: $2 f>\mu^{\prime}{ }_{s} c \tau / t_{0}$, where the characteristic diffusion time $\mathrm{t}_{0}$ is

$$
t_{0}=\frac{\lambda^{2}}{4 \pi^{2} D_{B}}=\frac{3 \eta a \lambda^{2}}{2 \pi k T}
$$

where $\mu_{s}$ is the reduced scattering coefficient of the medium, $\eta$ its viscosity, $a$ is the radius of the scattering particles and $c \tau$ the selected path length. As the quantity $\mu_{s}^{\prime} c \tau$ can be higher than 400 , the characteristic time $t_{0}$ has to be much higher than 1 second. Even using relatively big scatterers (with a diameter higher than $2 \mu m$ ), this implies a viscosity about 2 orders of magnitude higher than the viscosity of water. That is the reason why we used vegetal oil (olive oil), which has a very low absorption coefficient in the near infrared $\left(\sim 0.01 \mathrm{~cm}^{-1}\right)$ and a high viscosity $\left(84 \mathrm{mPa} . \mathrm{s}\right.$ at $\left.20^{\circ} \mathrm{C}\right)$. We 
adjusted the concentration of a suspension of oxide aluminum $\left(\mathrm{Al}_{2} \mathrm{O}_{3}\right)$ in order to get a reduced scattering coefficient $\mu_{s}^{\prime}=10 \mathrm{~cm}^{-1}$ (the size distribution of the scatterers is in the range $1-5 \mu \mathrm{m}$, the index of refraction of the oil is 1.47 while the index of $\mathrm{Al}_{2} \mathrm{O}_{3}$ is 1.76). The sample was filling a large $11 \mathrm{~cm}$ diameter cylindrical glass container, with a thickness of $4.5 \mathrm{~cm}$.

The time-resolved reflectance was measured using our system with a source-detector separation of $1 \mathrm{~cm}$. The incident light power was $7 \mathrm{~mW}$, and $5 \mathrm{~mW}$ after background substraction. The ensemble averaging was performed over 32000 modulation periods, with a 10min total acquisition time due to non-optimization of the acquisition system (residual problems in the A/D converters synchronization, calculation time...) but there is no doubt that the theoretical value (3min 30s) can be easily reached and even be decreased using a multiple detectors acquisition.

The same measurement was then performed in almost the same conditions, using a streak camera and a mode-locked Ti-Sa laser at $800 \mathrm{~nm}$. The integration time was 3min20s (20 acquisitions with an on-chip integration time of $10 \mathrm{~s})$. The incident light power was $5 \mathrm{~mW}$. Both results were fitted using Monte Carlo simulations taking into account the temporal response of the systems. The time origin was adjusted for each curve during the fitting procedure and the results were normalized. The values obtained for the optical coefficients are presented in table 1, while the experimental results are plotted in figure 2 (after adjustment of both time origin and normalization, using the results of the fitting procedure). We obtained a pretty good similarity between these results: this is the first experimental evidence of the feasibility of our interferometric setup for the measurement of the time-resolved reflectance on a liquid scattering medium. In order to underline the sensitivity of our setup, the signal level in figure 2 at $t=2000 \mathrm{ps}$ corresponds to a power of 50fW collected by the multimode detection fiber (within the $45 \mathrm{ps}$ time gate), that is to half a photon per acquisition in the detected mode, and we achieve a SNR of about 16 at this point. 
The next step now will be to increase the modulation frequency in order to work with suspensions in water, or with biological media. Application of formula (3) leads to a frequency of $10 \mathrm{kHz}$ for $1 \mu \mathrm{m}$ diameter microspheres in water. Concerning biological media, we have tested our interferometric setup on the forearm of a volunteer at rest, and the result is plotted in figure 3 , together with the result obtained using the streak camera. The pertinence of the optical coefficients obtained from these results (see table 2), that compare well with other works ${ }^{[5]}$, are encouraging features for the following. This result has of course to be counterchecked from tests carried out at higher frequencies, and from decorrelation measurements: this experiment was performed at rest, and since the blood flow increases by a factor of 30 during an exercise ${ }^{[10]}$, the modulation frequency will probably have to be increased by the same factor. For all these reasons, our aim is now to reach a modulation frequency of $10 \mathrm{kHz}$. As the main limitation of our system is the laser source, whose mechanical frequency modulation system cannot reach such a value, we have to look for other possibilities using for example DBR or DFB laser diodes.

In summary, we have proved the possibility to perform time-resolved measurement of the light scattered by a liquid medium using speckle interferometry. The results were compared to measurements performed with a femtosecond Ti-Sa laser and a streak camera, and a good correlation was obtained. The system was tested on the forearm of a volunteer, leading to interesting results which have to be confirmed by increasing the modulation frequency of the system.

We would like to thank Xavier Renon for his technical support in the development of the detection circuit. 


\section{References}

[1] M. Kathleem Alam, http://infoserve.sandia.gov/cgi-bin/techlib/accesscontrol.pl/2000/002584.pdf , Sandia report (2000), and references therein.

[2] Alessandro Torricelli, Valentina Quaresima, Antonio Pifferi, Giovanni Biscotti, Lorenzo Spinelli, Paola Taroni, Marco Ferrari and Rinaldo Cubeddu, Phys. Med. Biol. 49, 685 (2004)

[3] Robert J Hunter, Micheal S Patterson, Thomas J Farrell and Joseph E Haward, Phys. Med. Biol 47, 193 (2002)

[4] Adam Liebert, Heidrun Wabnitz, Jens Steinbrink, Hellmuth Obrig, Michael Möller, Rainer Macdonald, Arno Villringer, and Hert Rinneberg, Applied Optics 43, 3037 (2004)

[5] Alwin Kienle and Thomas Glanzmann, Phys. Med. Biol. 44, 2689 (1999)

[6] J.-M. Tualle, H.L. Nghiem, D. Ettori, R. Sablong, E. Tinet, and S. Avrillier, J. Opt. Soc. Am. A 21, 24 (2004)

[7] J.-M. Tualle, E. Tinet, S. Avrillier, Optics communication 189, 211 (2001)

[8] J.-M. Tualle, international patent WO0188507, november $22^{\text {nd }} 2001$

[9] D. J. Pine, D. A Weitz, P. M. Chaikin, and Herbolzheimer, Phys. Rev. lett. 60, 1134 (1988)

[10] H Monod, R Flandrois, Physiologie du sport, Editions Mason, Paris, Milan, Barcelone (1994). 


\section{Table caption}

Table 1 : Optical coefficients obtained on the liquid phantom.

Table 2: Optical coefficients obtained on the forearm of a volunteer. 


\section{Figures caption}

Figure 1: Experimental setup.

Figure 2: Comparison of the time-resolved reflectance measured on the liquid phantom using the interferometric setup (open circles) and using a streak camera (straight line).

Figure 3: Time-resolved reflectance measured on the forearm of a volunteer (using the interferometric setup (open circles), and using a streak camera (straight line). 


\begin{tabular}{|c|c|c|}
\hline & $\mu_{s}^{\prime}\left(\mathrm{cm}^{-1}\right)$ & $\mu_{a}\left(\mathrm{~cm}^{-1}\right)$ \\
\hline Interferometer & $10.3 \pm 0.1$ & $0.02 \pm 0.001$ \\
\hline Streak camera & $10.2 \pm 0.1$ & $0.02 \pm 0.001$ \\
\hline
\end{tabular}

Table 1 


\begin{tabular}{|c|c|c|}
\hline & $\mu_{s}^{\prime}\left(\mathrm{cm}^{-1}\right)$ & $\mu_{a}\left(\mathrm{~cm}^{-1}\right)$ \\
\hline Interferometer & $14 \pm 0.5$ & $0.18 \pm 0.005$ \\
\hline Streak camera & $14+0.4$ & $0.17+0.004$ \\
\hline
\end{tabular}

Table 2 


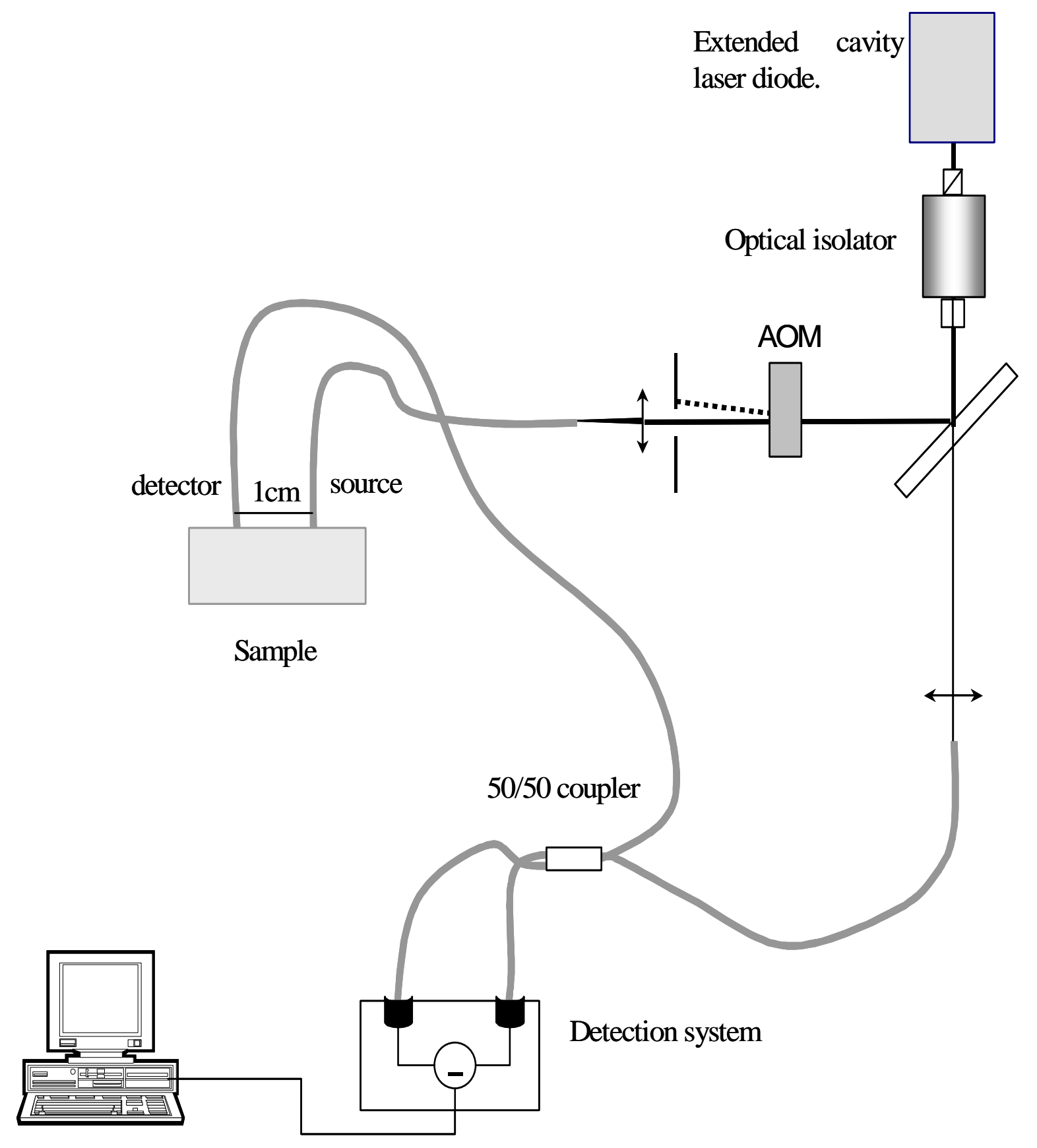

figure 1: 


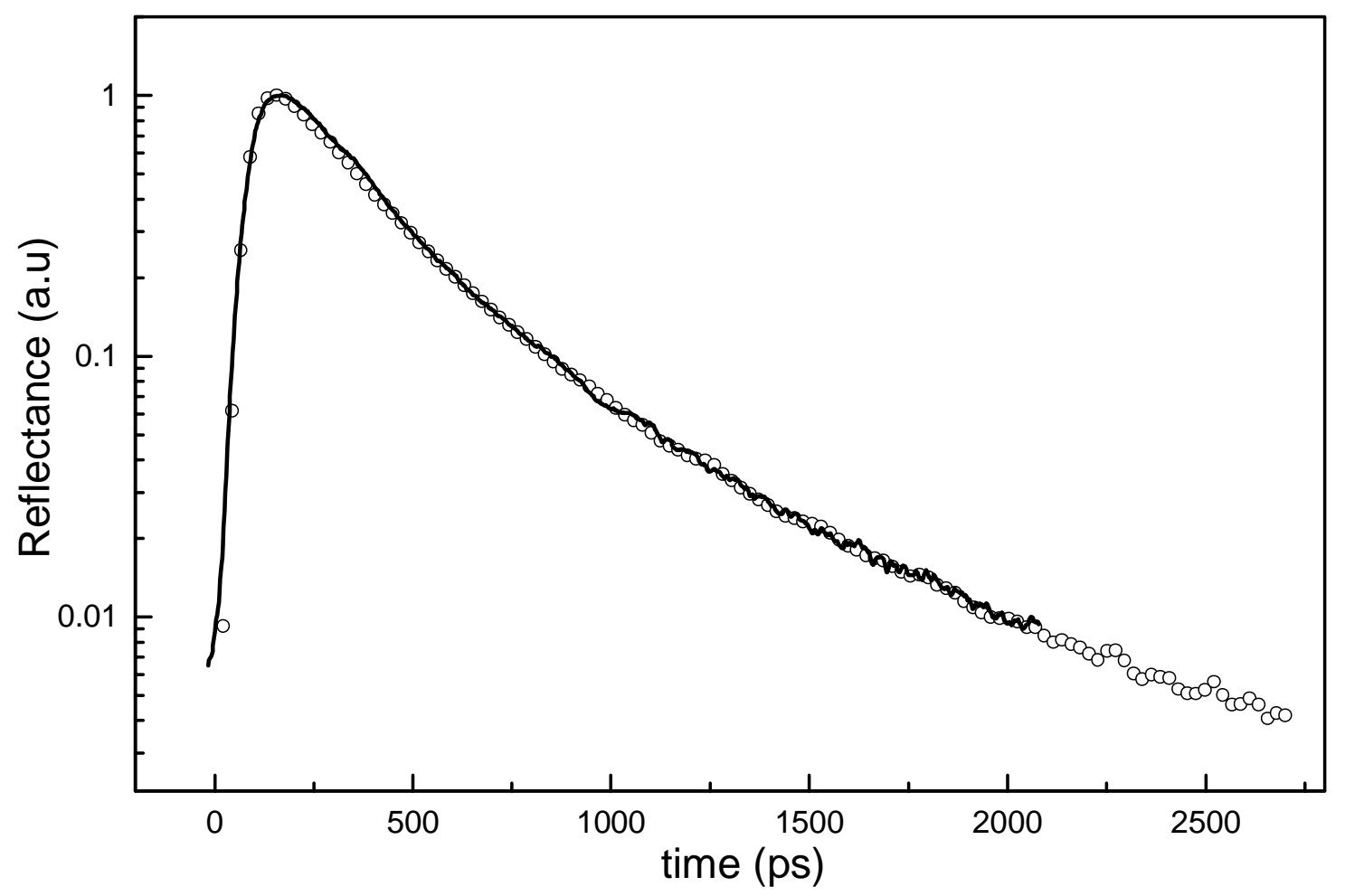

Figure 2 


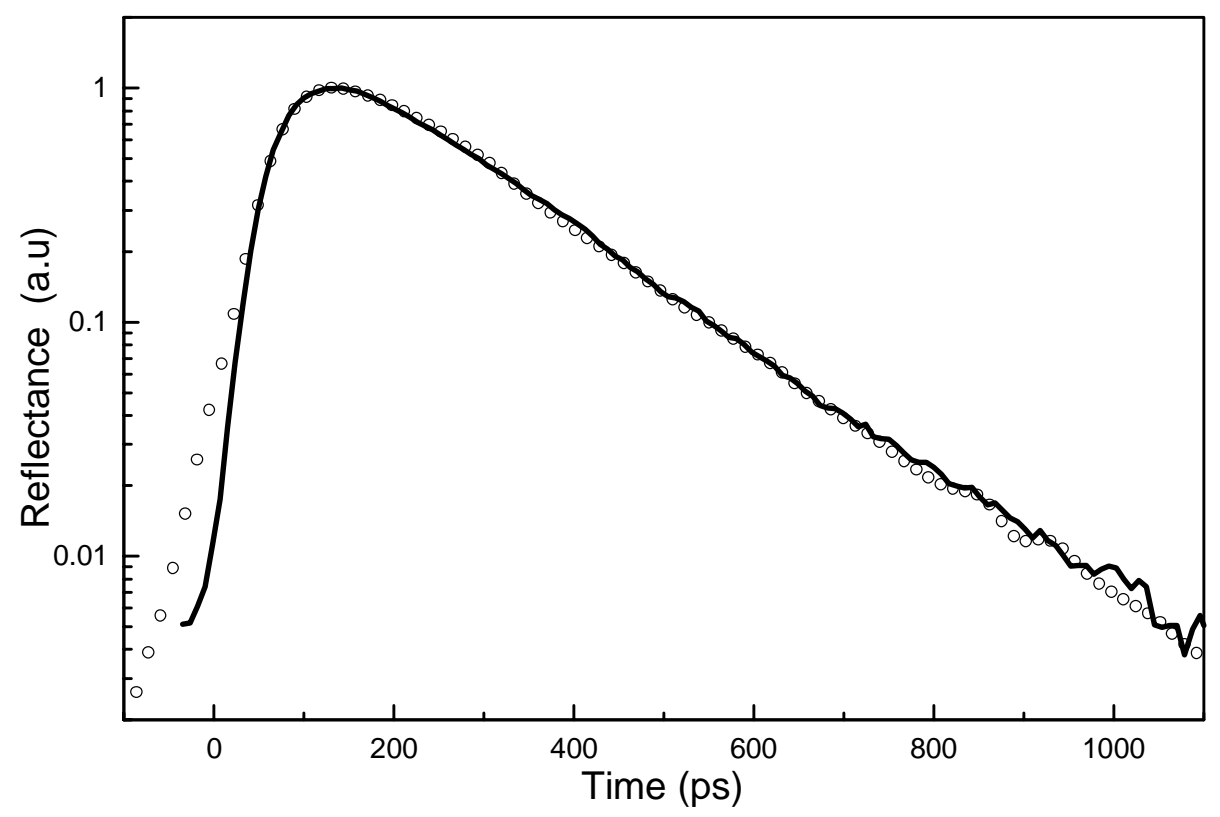

Figure 3 